\title{
L'orchestre : un milieu de convivence et d'éducation au sein des prisons
}

\author{
La orquesta: un lugar de convivencia y de educación en las cárceles
}

The orchestra: convivence and education within prisons

Accueil: 16/12/2019
Évaluation: 17/04/2020
Acceptation: $28 / 05 / 2020$
Article d'enquête
https://doi.org/10.19053/01227238.11921

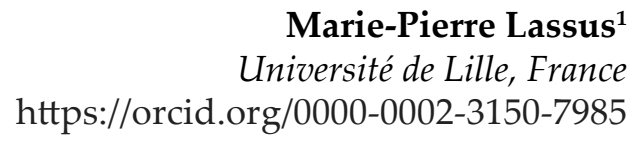

RÉSUMÉ

L'objectif est de montrer comment l'orchestre peut être un lieu d'éducation capable de redonner confiance et estime de soi aux personnes détenues et améliorer le vivre-ensemble dans les prisons. L'étude s'appuie sur la méthodologie de la recherche-action (qualitative-quantitative) et de la recherche-création pour montrer que les valeurs apprises dans l'orchestre (écoute, responsabilité, discipline, solidarité, convivence) sont applicables au quotidien et peuvent engager des processus de transformation en faisant découvrir à chacun des possibilités inédites qui facilitent la réinsertion dans la vie sociale. Dans le contexte des prisons françaises, caractérisées aujourd'hui par une surpopulation croissante générant conflits et violence, ou celui des prisons vénézuéliennes où ces problèmes sont récurrents, la création d'orchestres est un projet politique, visant à instaurer un espace commun et personnel où peuvent interagir les personnes détenues vouées en ces lieux à la séparation et à la fragmentation des espaces et du temps. En conclusion, l'éducation ne peut avoir lieu sans la création d'un milieu convivial (au sens donné par le mouvement international convivialiste) où s'affirment les principes d'individuation et d'opposition créatrice qui sont au fondement de toute véritable démocratie comme de l'orchestre conçu comme une micro-société.

Mots - clé : orchestre; milieu; prison ; éducation; convivialisme.

\section{RESUMEN}

El objetivo es mostrar cómo la orquesta puede ser un lugar de educación capaz de devolver la confianza y la autoestima a los detenidos y mejorar la convivencia en las cárceles. El estudio se basa en la metodología de investigación-acción (cualitativa-cuantitativa) y de investigación-creación para mostrar que los valores aprendidos en la orquesta (escucha, responsabilidad, disciplina, solidaridad,

1 Professeur titulaire en musicologie, Faculté d’Arts et Humanités, Université de Lille (France). Directrice du master international Art et Responsabilité Sociale. Courrier électronique : marie-pierre.lassus@univ-lille.fr. 
convivencia) son aplicables en la vida y pueden iniciar procesos de transformación introduciéndose entre sí en nuevas posibilidades que facilitan la reintegración a la vida social. En el contexto de las prisiones francesas, caracterizadas hoy por un hacinamiento creciente que genera conflictos y violencia, o el de las prisiones venezolanas donde estos problemas son recurrentes, la creación de orquestas es un proyecto político, dirigido a establecer un espacio común y personal donde los detenidos puedan interactuar en estos lugares dedicados a la separación y fragmentación de los espacios y tiempos. En conclusión, la educación no puede tener lugar sin la creación de un ambiente de convivencia (en el sentido dado por el movimiento internacional de Convivencia) donde se afirman los principios de individualización y oposición creativa, que están en la base de cualquier democracia, así como la orquesta concebida como una micro-sociedad.

Palabras clave: orquesta; medio ambiente; prisión; educación; convivencia.

\begin{abstract}
The aim is to show how the orchestra could be a convivence "milieu" and an education place that is able to restore confidence and self-esteem to convicts and improve the living together in prisons.

The study is based on the research-action methodology (qualitative-quantitative) and the research-creation to show that the learned values inside the orchestra (listening, responsibility, discipline, solidarity, convivence), can be apply in everyday's life and can initiate a processus of change by introducing each other to new possibilities that facilitate reintegration into social life. In the context of French prisons characterised nowadays by a growing overcrowding causing conflicts and violence, or within
\end{abstract}

ABSTRACT

Venezuelan prisons where these problems are repeated, the creation of orchestra is a political project aimed to establish a personal and community aera where convicts can interact in these places dedicated to separation and fragmentation of spaces and time.

In conclusion, education cannot take place without the creation of a friendly "milieu"(in the meaning given by the international convivialist movement) where the principles of individualization and creative opposition are asserted, which are at the foundation of any true democracy as well as the orchestra conceived as a micro-society.

Key words : orchestra; "milieu"; prison; education; convivialism.

\title{
INTRODUCTION
}

L'objectif est de montrer comment l'orchestre participatif dans les prisons (et ailleurs) peut être un lieu d'éducation capable de redonner confiance et estime de soi aux personnes détenueset contribuer à l'amélioration de leurs conditions de vie, par la création d'un milieu convivial. Après avoir évoqué l'univers carcéral comme lieu de séparation des mondes, je mettrai en regard le cas des prisons françaises, caractérisées aujourd'hui par une surpopulation croissante, génératrice de conflits, avec celles du Venezuela où la violence est récurrente. La création,depuis 2008, des orchestres et deschoeurs pénitentiaires par El Sistema ${ }^{2}$

2 Adopté par l'Etat comme politique nationale, El Sistema,connu sous le nom de El Sistema de Coros y Orchestas Juveniles e Infantiles 
vénézuéliena eu pour effet de la réduire en donnant des possibilités de réinsertion aux détenus. Je prendrai l'exemple de ce programme social de la musique, inclus par L'UNESCO dans le projet interdisciplinaire Hacia una Cultura de Paz, en 1995 et, en 1999, lors de la Conférence Générale à Paris, en faveur de l'éducation artistique et de la créativité pour la construction d'une culture de paix, comme référencehistorique d'inclusion sociale et de participation communautaire. Par la suite, plus de vingt - cinq pays ont cherché à adapter à leurs contextesce modèle j'ai moi-même adapté à l'université de Lille (France) et sa région.

\section{La prison : lieu de l'altérité et de la violence}

Pour la plupart des citoyens, la prison est une zone d'ombre qui marque une ligne de partage entre deux mondes : ceux qui participent à la vie en société et les autres qui en sont exclus, vivant dans un monde à part, étranger à la vie civile mais qui n'en reste pas moins un lieu de vie.Considérée abstraitement la prison est toujours pour lesautres, radicalement autres, coupés du monde et de la société. Elle a pour effet de produire de l'altérité en créant une frontière entre deux sortes d'humains. Pénétrer dans ce lieu exige de tout laisser, et d'abord, son identité. D'emblée, le prisonnier comme le citoyen est mis à l'épreuve et doit s'ouvrir à l'étrangeté de ce mondesingulier qui se présente concrètement comme une succession de portes à franchir comme autant de frontières séparant les espaces et les êtres :

Ces espaces sont reliés entre eux par des circulations dans lesquelles les détenus ne font que passer pour aller d'une micro-prison à une autre, sous le contrôle, direct ou indirect, des surveillants. Aussi, il n'y a pas d'espace dans la prison qui ne réponde à un usage spécifique, l'entre-deux, la frange, n'a pas d'existence propre... ${ }^{3}$.

D'après Hanna Arendt, il n'y a de "monde » que commun, formé par une " pluralité d'individus singuliers », condition même du politiquequi se définit selon elle par les relations que les humains établissent entre eux. "Le monde, comme tout entre-deux, relie et sépare en même temps les hommes $»^{5}$, affirme $\mathrm{H}$. Arendt pour qui vivre, sentir, agir, partager, actes qui sont au fondement de notre existence au monde, ont pour point commun de nous mettre à l'écoute de cet entre-deux. À cet égard, la prison se présente comme un «antimonde $»^{6}$, un

\footnotetext{
de Venezuela constituye un exemple de programme social et artistique. Créé en 1975 par le vénézuélien José-Antonio Abreu (19392018), musicien et économiste, ce programme d'éducation musicale, rendu accessible à tous les jeunes par la gratuité de l’enseignement et des instruments (de grande qualité) qui leur sont donnés, se maintient encore aujourd'hui malgré la situation économique du pays qui compte une majorité de jeunes. En 2015, le Venezuela était parsemé d'orchestres: 285 de la Petite Enfance (entre 4 et 6 ans); 220 orchestres d'Enfants (entre 7 et 16 ans), 180 de Jeunes (entre 16-22 ans) 30 orchestres professionnels, 360 groupes choraux, 1355 groupes choraux affiliés et 15000 professeurs (ainsi que 2à ateliers de lutherie).

3 Didier Cholet (dir), Les nouvelles prisons. Enquête sur le nouvel univers carcéral français. (Rennes : PUR, 2015 ), 218.

4 Hannah Arendt,Qu'est-ce que la politique? Texte établi par Ursula Ludz. (Paris : Seuil, 1995), 42.

6 Olivier Milhaud Olivier et Marie Morelle, L La prison entre monde et antimonde ». Géographie et culture. (57), (Paris : L’Harmattan,
}

5 Ibid. 
«trou noir », le produit d'une société « qui prend part à l'édification d'un centre et de marges sociaux et spatiaux ». Il s'agit d'un lieu " articulé aux réseaux de la marginalité et aux systèmes de contrôle et de régulation $»^{7}$.En prison il $n^{\prime} y$ a pas d'entre-deux : tout est fait pour qu'il n'y en ait pas ; ce qui rend ces lieux inhumains : "Arrivé en prison, on perd son statut d'humain » affirment les détenus qui en font l'expérience dès leur première entrée dans ces lieux. Cela commence par l'architecture : dans les nouvelles constructions, cellules, ateliers, salles de classe, cours de promenade sont des prisons dans les prisons. La «population » carcérale est répartie en unités visant à séparer les hommes des femmes et ceux jugés les plus «dangereux », mis momentanément en " quartier d'isolement ». Mais il y a plus. L'espace manque aux détenus, confinés dans des cellules où ils ne disposent pas du minimum vital : tel est le constat de la Cour européenne des droits de l'homme qui vient de condamner la France pour avoir violé l'article 3 de la convention européenne selon lequel « nul ne peut être soumis à la torture ni à des peines ou traitements inhumains dégradants ». Au $1^{\text {er }}$ octobre 2019, les prisons françaises contenaient 70818 détenus pour 61065 places, avec, dans 48 établissements, une densité supérieure allant de 150\% à 200\% (pour sept d'entre elles). Le droit de recours ayant été prévu dans l'article 13 de cette convention, la France est condamnée aujourd'hui à indemniser les 32 plaignants ${ }^{8}$ pour le manque d'espace $\left(3 \mathrm{~m}^{2}\right)$ subi pendant toute la durée de leur peine (sur plusieurs années) et les conditions indignes d'habitation (matelas au sol avec parfois la présence de punaises, rats ou cafards), les privant de tout accès à l'enseignement, aux soins et à la cour de promenade. Il est imposé aujourd'hui à la France de résoudre le problème et de permettre l'accès aux activités de formation et de travail à tous les détenus. Considérée par la Cour européenne comme un « traitement inhumain », la surpopulation carcérale a des conséquences dans l'organisation de la vie quotidienne en prison ${ }^{9}$.Même si nous n'avons pas connu ces situations extrêmes lors de nos interventions dans les onze prisons du Nord de la France, nous avons pu observer des conditions de vie difficiles en maison d'arrêt spécialement où il n'est pas rare de trouver plusieurs personnes vivant dans une cellule de $9 \mathrm{~m}^{2}$. Comment intervenir dans ce contexte et avec quel(s) moyens ? En tant que communauté dont la caractéristique est de se former dans le double objectif de s'accorder et de " s'opposer sans se massacrer» ${ }^{10}$, l'orchestre peutpermettre de retrouver une certaine convivence et proposer une

2006), 11.

7 Ibid.

8 Issus des prisons de Ducos (Martinique) Faa' Nuutania (Polynésie française), Baie-Mahault (Gaudeloupe), Nice et Fresnes (Val de Marne). Dans la Maison d'arrêt de Toulouse-Seysses,271 matelas au sol ont été installés en juillet 2019, tandis que le taux d’occupation de celle de Nîmes sélève à $200 \%$. Source : Jean-Baptiste Jacquin, «Surpopulation carcérale : la France condamnée, Le Monde $1^{\text {er }}$ février 2020.

9 Par exemple, les détenus sont obligés d’attendre deux ou trois mois pour pouvoir se rendre à l'activité sport, une activité très prisée par les hommes en milieu carcéral. Cette attente peut aussi concerner la mise à exécution d'une sanction (quartier d'isolement ou quartier disciplinaire), sans parler de l'augmentation de travail du surveillant en charge de 120 à 130 détenus par journée dans les maisons d'arrêt de Toulouse et de Nîmes notamment.

10 Selon la formule de Marcel Mauss qui définit ici le politique : "C’est ainsi que demain, dans notre monde dit civilisé, les classes et les nations et aussi les individus, doivent savoir sopposer sans se massacrer et se donner sans se sacrifier les uns aux autres ",Marcel, Mauss, Essai sur le don : forme et raison de léchange dans les sociétés archaïques. Sociologie et anthropologie. (Paris : PUF, 1950). 
réponse à la question de la violence et de la réinsertion, l'une des principales missions de ces établissements, selon la loi pénitentiaire du 24 novembre $2009^{11}$. Il y est affirmé la nécessité de préparer le retour des détenus dans la société dèsla prison, considérée comme lieu d'apprentissage, de formation et de travail. Maiscomment résoudre ce paradoxe de la réinsertion par l'enfermement en France quand on sait queles conseillers pénitentiaires d'insertion et de probation (CPIP), en charge d'une multitude de dossiers (environ 80 à 130 dossiers chacun) ne peuvent pas, à eux seuls, répondre à cette mission ? Comment concilier éducation et punition, travail éducatif et discipline $?^{12} S^{\prime}$ il est admis, en théorie, que « la finalité de la prison ne se résume pas à l'expiation mais implique la transformation $d u$ détenu $»^{13}$, celle-ci ne peut s'accomplir que dans un milieu propice, grâce à l'accueil des citoyens. Le système pénitentiaire espagnol offre aux personnes détenues et en particulier aux femmes ${ }^{14}$, victimes le plus souvent de violences, des programmes spécifiques afin de leur donner accès à une éducation (élémentaire, secondaire ou supérieure) susceptible d'améliorer leurs conditions de vie, liées à la pauvreté (plus importante que chez les hommes). Elles peuvent ainsi trouver en prison les moyens de remédier à cette carenceéducative et profiter de ce temps d'incarcération pour se former. L'étude réalisée par Fanny Añaños dans les prisons d'Espagne montre que 84,3\% d'entre elles participent à ces programmes de formation et peuvent ainsi initier un processus de changement. Même si le niveau d'instruction reste faible en général, la mise en place de ces programmes et les interventions socioéducatives les aident à sortir de leurs conditions de vulnérabilité. D'après cette étude, l'éducation en prison serait une voie possible de transformation chez les personnes détenues qui peuvent ainsi préparer leur sortieen développant les capacités nécessaires pour mener une vie créatrice et responsable ${ }^{15}$.Si l'on admet que tout conflit, dès lors qu'il est conscientisé, peut mener à des processus de changement, il est possible de concevoir la prison comme un espace d'éducation et de socialisation. Celui-ci a pris la forme d'orchestres et de choeurs au Venezuela, dont le but visé par son créateur, $\mathrm{K}-\mathrm{L}$ Mora Aragon ${ }^{16}$, est la transformation des conduites et la réinsertion sociale.

11 Loi du 24 novembre 2009, article $1:$ : le régime d'exécution de la peine de privation de liberté concilie la protection de la société, la sanction du condamné et les intérêts de la victime avec la nécessité de préparer l'insertion ou la réinsertion de la personne détenue afin de lui permettre de mener une vie responsable...".

12 Chantraine, Gilles et Sallé Nicolas. Eduquer et punir. Travail éducatif, sécurité et discipline en établissement pénitentiaire pour mineurs. Vol: $54 . \mathrm{N}^{\circ} 3$ (2013).

13 Cesare Beccaria, Des délits et des peines. Traduction de Maurice Chevallier. Préface de Robert Badinter (Paris : Flammarion, 1991, [Genève 1965]), 23.

14 Même si celles-ci ne représentent que 7,64 \% de la population pénitentiaire, ce taux est le plus élevé en Europe/

15 Añaños-Bedriñana, F.T., \& García-Vita, M.M. (2017. ¿Desarrollo humano en contextos punitivos? Análisis Socioeducativa desde las vulnerabilidades sociales y el género. Revista Criminalidad, 59 (2): 109-124.

16 Musicien vénézuélien (corniste), membre de l'orchestre Simon Bolivar, Kleiberth Lenin Mora Aragon est aussi avocat, diplômé en Droit international et humanitaire et en criminologie. Il est actuellement doctorant de l'université de Lille. 


\section{Les orchestres pénitentiaires au Venezuela}

La violence générée par les conflits dans les prisons vénézuélienne a été atténuée de 2007 à 2017 grâce à la création de ce programme musical issu de El Sistema ciblantune meilleure utilisation, constructive, du temps passé en détention. Le but de ce programme était de promouvoir à travers l'apprentissage musical, une (ré) éducation, de valeurs sociales et existentielles par une méthodologie centrée sur la participation active. Pratiquée de manière intensive (de $8 \mathrm{H}$ à $16 \mathrm{H}$ du lundi au vendredi) la musique est répartie au quotidien entre cours collectifs et cours individuels en vue des répétitions préparant aux concertsintramuros et hors les murs. C'est ainsi que chaque année, en décembre se réunissent les prisonniers des huit prisons, pour jouer authéâtre Teresa Carreño, (l'une des plus grandes salles de concert de la ville de Caracas) où ils sont applaudis par les citoyens venus (nombreux) les écouter. Le processus d'apprentissage intensif et ininterrompu permet de faire gagner rapidement en dextérité les détenus, qui peuvent ainsi accroître,en même temps que leur niveau technique,leur auto-estime par le sentiment d'exister de se sentir « capables ». Car ne sont pas seulement des capacités musicales qui sont visées dans ce processus sinonla transformation de la personne et de sa conduite envers les autres et envers lui-même. Le respect et l'écoute, le sens de la responsabilité (de l'instrument attribué comme de sa présence régulière à l'orchestre) et de la solidarité acquis par le travail d'équipe, fait de ce programme, un véritable projet de vie développant l'autonomie et la prise de décision, nécessaires à tout citoyen. La musique, en tant que pratique relationnelle et non verbale, devient un mode d'existence facilitant les relations avec autrui que l'on apprend à respecter et à encourager pour l'effort accompli. Comme le dit Kleibert Lenin Mora Aragon, l'initiateur de ce projet, « nous ne cherchons pas tant à former des musiciens que des citoyens susceptibles de se réinsérer dans la société de manière durable $»^{17}$. De fait son étude montre que parmi les 8000 personnes ayant participé à l'orchestre pénitentiaire seulement $10 \%$ d'entre elles ont récidivé. Visant au début les $10 \%$ de la population carcérale, ce chiffre a été largement dépassé dès le début du projet où l'on comptait $30 \%$ voire $90 \%$ de volontaires dans certaines prisons. Par ailleurs, il arrive que d'anciens détenus soient intégrés à leur sortie dans les orchestres de El Sistema en raison de leur haut niveau musical. Quant aux autres ils peuvent également devenir musiciens et intégrer des écoles de musique ou conservatoires pour y enseigner.Dans ce projet, l'accent est mis sur la relation entre le comportement musical et le comportement social et individuel, le premier pouvant agir de façon bénéfique sur le second. Pour pouvoir en rendre compte, des questionnaires ont été distribués aux participants et des entretiens ont eu lieu avec des détenus et des ex-détenus, des surveillants, les familles, ainsi que les enseignants. Les résultats obtenus entre 2007 - 2018 sont reportés dans le tableau ci-joint où l'on peut voir apparaître à gauche les indicateurs de valeurs à acquérir dans l'orchestre

17 Mora Aragon, Kleiberth Lenin, Trascendencia de la conducta a través de la práctica orquestal en ambientes poco gratificantes. Informe técnico (2007-2014), 5 . 
(responsabilité et discipline, respect, motivation, auto-estime, émotions négatives et positives, engagement, communication et solidarité) avec les questions correspondantes tandis que les objectifs apparaissent dans la colonne de droite en regard de l'auto-évaluation des détenus.

Significatifs sont les résultats concernant l'auto-évaluation montrant que $94,25 \%$ parvenaient à des limites jamais atteintes de leurs possibilités, tandis que plus de la moitié affirmait avoir accompli, grâce à ce projet, l'accord de " convivence ». Le sens de la responsabilité, la discipline, le respect, l'autoestime, l'engagement, le travail d'équipe, la tolérance et la façon de gérer ses émotions, ont été apprises dans l'orchestre devenu un lieu d'éducation à la vie tant individuelle que collective. $100 \%$ ont répondu positivement à la satisfaction procurée par cette pratique qui, pour $100 \%$ a permis de renforcer leur identité en répondant à leur besoin vital d'expression.Ayant pu me rendre au Venezuela pour les voir travailler, j'ai pu constaterl'acquisition de ces valeurs, tant dans les orchestres juvéniles et infantiles que dans les prisons vénézuéliennesoù les personnes détenues témoignaient volontiers de leur joie de jouer et de se découvrir des capacités ignorées grâce à cette pratique musicale exigeante qui mobilise un travail sur soi, autrement dit une éthique susceptible de les transformer ${ }^{18}$.

\section{TABLA 1. CUESTIONARIO APLICADO A LOS PRIVADOS DE LIBERTAD 2007 - MARZO 2018}

\begin{tabular}{|c|c|c|c|c|c|c|c|c|}
\hline \multirow[b]{2}{*}{$\begin{array}{l}\text { DIMEN- } \\
\text { SIÓN }\end{array}$} & \multirow[b]{2}{*}{$\begin{array}{l}\text { INDI- } \\
\text { CADORES }\end{array}$} & \multirow{2}{*}{$\begin{array}{c}\text { VALORACIÓN } \\
\text { REALIZADA POR EL } \\
\text { DOCENTE ITEMS/ } \\
\text { INDICADORES } \\
\end{array}$} & \multicolumn{2}{|c|}{$\sum \mathrm{S}+\mathrm{CS}$} & \multirow[b]{2}{*}{$\begin{array}{l}\text { AUTOVALORACIÓN } \\
\text { INTERNO ITEMS/ } \\
\text { INDICADORES }\end{array}$} & \multicolumn{2}{|c|}{$\sum \mathrm{S}+\mathrm{CS}$} & \multirow[b]{2}{*}{$\begin{array}{l}\text { OBJETIVO } \\
\text { OPERA- } \\
\text { TIVO }\end{array}$} \\
\hline & & & Fs & $\%$ & & Fs & $\%$ & \\
\hline \multirow{7}{*}{ 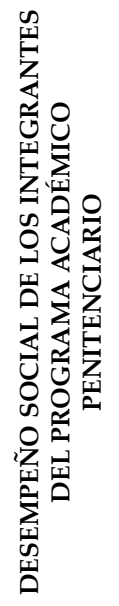 } & \multirow{3}{*}{$\begin{array}{l}\text { RESPONSA- } \\
\text { BILIDAD } \\
\text { Y } \\
\text { DISCIPLINA }\end{array}$} & $\begin{array}{l}\text { 2. ¿Asiste puntualmente } \\
\text { al horario indicado? }\end{array}$ & 346 & 86.5 & $\begin{array}{l}\text { 2. ¿Asiste puntualmente } \\
\text { al horario indicado? }\end{array}$ & 393 & 98.3 & Credibilidad \\
\hline & & $\begin{array}{l}\text { 4. ¿Cumple con agrado } \\
\text { las órdenes? }\end{array}$ & 351 & 87.8 & $\begin{array}{l}\text { 4. ¿Cumple con agrado } \\
\text { las órdenes? }\end{array}$ & 372 & 93.0 & Obediencia \\
\hline & & $\begin{array}{l}\text { 14. ¿Asume sus } \\
\text { responsabilidades? }\end{array}$ & 348 & 87.0 & $\begin{array}{l}\text { 15. ¿Asume sus } \\
\text { responsabilidades? }\end{array}$ & 391 & 97.8 & Reflexionar \\
\hline & RESPETO & $\begin{array}{l}\text { 13. ¿Es respetuoso frente } \\
\text { a las decisiones de los } \\
\text { demás? }\end{array}$ & 323 & 80.8 & $\begin{array}{l}\text { 14. ¿Es respetuoso frente } \\
\text { a las decisiones de los } \\
\text { demás? }\end{array}$ & 386 & 96.5 & Reconocer \\
\hline & $\begin{array}{l}\text { MOTIVA- } \\
\text { CION }\end{array}$ & $\begin{array}{l}\text { 10. ¿Es dinámico en las } \\
\text { actividades? }\end{array}$ & 373 & 93.3 & $\begin{array}{l}\text { 11. ¿Es dinámico en las } \\
\text { actividades? }\end{array}$ & 360 & 90.0 & Impulso \\
\hline & \multirow[t]{2}{*}{$\begin{array}{l}\text { AUTOES- } \\
\text { TIMA }\end{array}$} & $\begin{array}{l}\text { 6. ¿Es cuidadoso de su } \\
\text { presentación personal? }\end{array}$ & 381 & 95.3 & $\begin{array}{l}\text { 6. ¿Es cuidadoso de su } \\
\text { presentación personal? }\end{array}$ & 390 & 97.5 & Amor propio \\
\hline & & 8. ¿Es creativo? & 310 & 77.5 & 8. ¿Es creativo? & 323 & 80.8 & Autovalor \\
\hline
\end{tabular}

18 J'ai pu constater dans ma propre expérience de l’orchestre participatif en France que certains détenus avaient cessé de prendre des médicaments ou autres substances pour pouvoir être présents et actifs à lorchestre. 


\begin{tabular}{|c|c|c|c|c|c|c|c|c|}
\hline \multirow{14}{*}{ 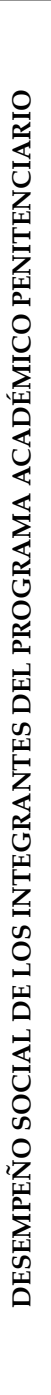 } & \multirow{4}{*}{$\begin{array}{l}\text { EMOCIONES } \\
\text { NEGATIVAS }\end{array}$} & $\begin{array}{l}\text { 3. ¿Expresión facial } \\
\text { rígida? }\end{array}$ & 41 & 10.3 & & & & $\begin{array}{l}\text { Reducción } \\
\text { de bienestar }\end{array}$ \\
\hline & & $\begin{array}{l}\text { 7. ¿Maneja un vocabu- } \\
\text { lario rudo? }\end{array}$ & 18 & 4.5 & & & & Violencia \\
\hline & & $\begin{array}{l}\text { 15. ¿Se altera ante una } \\
\text { crítica? }\end{array}$ & 23 & 5.75 & $\begin{array}{l}\text { 16. ¿Es violento ante } \\
\text { una crítica? }\end{array}$ & 18 & 4.50 & $\begin{array}{l}\text { Reactividad } \\
\text { emocional }\end{array}$ \\
\hline & & $\begin{array}{l}\text { 17. ¿Habla de su } \\
\text { entorno familiar? }\end{array}$ & 82 & 20.5 & & & & $\begin{array}{l}\text { Dificultad } \\
\text { afectiva }\end{array}$ \\
\hline & \multirow{3}{*}{$\begin{array}{l}\text { EMOCIONES } \\
\text { POSITIVAS }\end{array}$} & $\begin{array}{l}\text { 1. ¿Es preocupado en } \\
\text { hacer bien las tareas } \\
\text { encomendadas? }\end{array}$ & 325 & 81.5 & $\begin{array}{l}\text { 1. ¿Es preocupado en } \\
\text { hacer bien las tareas } \\
\text { encomendadas? }\end{array}$ & 375 & 93.8 & $\begin{array}{l}\text { Reconsi- } \\
\text { deración } \\
\text { cognitiva }\end{array}$ \\
\hline & & 16. ¿Es tolerante? & 331 & 82.8 & 17. ¿Es tolerante? & 301 & 86.5 & Aceptación \\
\hline & & & & & $\begin{array}{l}\text { 18. ¿Habla de su } \\
\text { entorno familiar? }\end{array}$ & 295 & 73.8 & $\begin{array}{l}\text { Reinterpreta- } \\
\text { ción }\end{array}$ \\
\hline & \multirow{3}{*}{$\begin{array}{l}\text { PERTI- } \\
\text { NENCIA Y } \\
\text { COMPRO- } \\
\text { MISO }\end{array}$} & $\begin{array}{l}\text { 11. ¿Es cuidadoso con el } \\
\text { manejo de instrumentos } \\
\text { de trabajo? }\end{array}$ & 383 & 95.8 & $\begin{array}{l}\text { 12. ¿Es cuidadoso con el } \\
\text { manejo de instrumentos } \\
\text { de trabajo? }\end{array}$ & 390 & 97.5 & Conciencia \\
\hline & & & & & $\begin{array}{l}\text { 3. ¿Le produce } \\
\text { satisfacción el éxito de } \\
\text { la Orquesta? }\end{array}$ & 400 & 100 & Cohesión \\
\hline & & & & & $\begin{array}{l}\text { 9. ¿Considera como } \\
\text { suyos los éxitos obte- } \\
\text { nidos por la Orquesta } \\
\text { Sinfónica Penitenciaria? }\end{array}$ & 400 & 100 & $\begin{array}{l}\text { Fortalecer } \\
\text { la identidad } \\
\text { personal }\end{array}$ \\
\hline & \multirow{3}{*}{$\begin{array}{l}\text { COMUNICA- } \\
\text { CIÓN }\end{array}$} & $\begin{array}{l}\text { 5. ¿Es atento con la } \\
\text { gente que le solicita } \\
\text { información? }\end{array}$ & 352 & 88.0 & $\begin{array}{l}\text { 5. ¿Es atento con la } \\
\text { gente que le solicita } \\
\text { información? }\end{array}$ & 300 & 75.0 & $\begin{array}{l}\text { Interactuar } \\
\text { positivo }\end{array}$ \\
\hline & & & & & $\begin{array}{l}\text { 7. ¿Maneja un } \\
\text { vocabulario adecuado a } \\
\text { la institución? }\end{array}$ & 380 & 95 & $\begin{array}{l}\text { Respuesta } \\
\text { coherente al } \\
\text { entorno }\end{array}$ \\
\hline & & $\begin{array}{l}\text { 9. ¿Pregunta cuándo } \\
\text { presenta duda? }\end{array}$ & 346 & 86.5 & $\begin{array}{l}\text { 10. ¿Pregunta cuándo } \\
\text { desconoce un procedi- } \\
\text { miento? }\end{array}$ & 365 & 91.3 & $\begin{array}{l}\text { Informa- } \\
\text { ción con } \\
\text { propósito }\end{array}$ \\
\hline & $\begin{array}{l}\text { SOLIDA- } \\
\text { RIDAD }\end{array}$ & $\begin{array}{l}\text { 12. ¿Es solidario con sus } \\
\text { compañeros? }\end{array}$ & 351 & 87.8 & $\begin{array}{l}\text { 13. ¿Es solidario con sus } \\
\text { compañeros? }\end{array}$ & 346 & 86.5 & Unidad \\
\hline
\end{tabular}

Fuente: Mora Aragon K.L., Trascendencia de la conducta a través de la práctica orquestal en ambientes poco gratificantes. Informe técnico (2007-2018).

Dans cette expérience,K-L Mora Aragon a fait le lien entre comportement musical et comportement individuel/social. Il a pu vérifier son hypothèse selon laquelle l'implantation d'orchestres dans les lieux de conflit et de violence peut être un facteur de réinsertion sociale et de transformation de la personne. Ce fut à l'origine de mon travail à Lille (France) où j'ai développé une autre approche de l'orchestre, fondée sur la participation de citoyens musiciens et non-musiciens pour créer un espace commun au sein des prisonset permettre la rencontre entre les mondes à travers la pratique musicale et le jeu. Ce projet est aujourd'hui intégré dans une formation que j'ai créée à l'université de Lille. 


\section{L'orchestre participatif à Lille (France)}

Fondé sur la participation de citoyens bénévoles (étudiants, enseignants et chercheurs de toutes disciplines)l'orchestre participatif, ouvert aux musiciens et aux non-musiciens,a été créé et pratiqué depuis 2008 à l'université de Lille, dans le cadre du master Art et Responsabilité Sociale (ARS) que je dirige et, pendant trois ans, dans le programme Chercheurs Citoyens Le Jeu d'Orchestre, (2011 - 2014), financé par le Conseil Régional du Nord, en collaboration avec l'association Hors-Cadre ${ }^{19}$.

Dans des lieux voués à la fragmentation et à la séparation des espaces comme le sont les prisons, rassemblerpendant des journées entières et parfois pour deux semaines, des personnes inconnues au sein d'un même lieu pour y créer, grâce à l'orchestre, un milieu convivial, est un projet politique (au sens de H. Arendt et M. Mauss). L'occupation de l'espace (le gymnase, généralement) et du temps, celui, imprévisible, de la musique, contre la routine et l'emploi du temps (particulièrement contraint dans les Etablissements Pénitentiaires pour Mineurs), reste un défi.Pour qu'une rencontre puisse avoir lieu entre des mondes aussi différents que celui de la prison et celui de l'université, il faut quitter son identité (de chercheur, d'étudiant, d'enseignant ou de détenu) et abandonner tous préjugés pour pouvoir s'ouvrir à l'altérité : tel est l'enjeu d'un effort collectif qui nous place dans une posture de non-savoir ${ }^{20}$, considérée à la fois comme une éthique et une méthodologie dans les projets que je mène à Lille. Je précise que le non-savoir, loin d'être une ignorance, requiert au contraire un dépassement difficile de la connaissance pour pouvoir créer une relation d'égalité entre les participants, tous venus pour jouer, sans autre finalité.

\section{Le jeu et le non-savoir : une posture éthique}

En tant qu'activité ayant sa fin en soi ${ }^{21}$ et dont la fonction sociale a été décrite par Johan Huizinga, le jeu est central dans notre projet dont le but n'est pas d'apprendre la musique ni de l'utiliser à des fins thérapeutiques mais d'instaurer une dynamique, où s'affirme la liberté et lele plaisir de jouer. Cette action, précise Huizinga, s'accompagne d'un sentiment de tension et de joie, de conscience d'être " autre » que dans la vie quotidienne ${ }^{22}$. Jouer au sein de l'orchestre conduit à établir des relations de groupe qui permettent à chacun d'éprouver le sentiment d'exister en tant que sujet vivant et agissant. Ainsi, le jeu apparaît

19 Marie-Pierre Lassus (dir.), Le Jeu d'Orchestre. Recherche-action dans les lieux de privation de liberté.(PUL : 2015). Entre 2011 et 2014 le projet s'est décliné dans les onze Etablissements Pénitentiaires de la Région Nord Pas de Calais, au sein des Centres Pénitentiaires de Lille Annœullin, Lille Sequedin, Longuenesse et Maubeuge, au sein du Centre de Détention de Bapaume (Quartier Hommes et Quartier Femmes), au sein des Maisons d’Arrêt d’Arras, Béthune, Douai, Dunkerque et Valenciennes (Quartier Hommes et Quartiers Femme) et au sein de l'Etablissement Pénitentiaire pour Mineurs de Quiévrechain.

20 Marie-Pierre Lassus, Le non-savoir, paradigme de connaissance.(Louvain-Paris : EME,L'Harmattan, 2019).

21 Johann Huizinga, Homo ludens, Essai sur la fonction sociale du jeu.Traduit du néerlandais par Cécile Seresia, (Paris : Gallimard, 1988), 34-35. « Le jeu est une activité volontaire qui se développe à l'intérieur de limites temporelles et spatiales déterminées, selon des règles librement consenties mais indispensables".

22 Ibid. 
en réalité comme une quête de soi, une façon de se chercher en se perdant et en se retrouvant sans cesse, pouvant mener à une véritable création de soi et du monde ${ }^{23}:$ " c'est en jouant et peut-être seulement quand il joue que l'enfant ou l'adulte est libre de se montrer créatif et de découvrir le soi " affirme le psychanalyste D-W Winnicott ${ }^{24}$ pour qui le jeu est universel et correspond à la santé.Selon lui l'art est un jeu et si les humains jouent, c'est pour des raisons vitales. Cela permet deretrouver une vie créatrice c'est-à-dire " le fait de ne pas être tué, annihilé continuellement par soumission ou par réaction au monde qui empiète sur nous » et « le fait de porter sur les choses une regard toujours neuf $»^{25}$.Cette relation entre jeu et liberté avait déjà été observée par le poète Novalis (1772 - 1801) : « [...] c'est dans le jeu seulement que l'homme prend véritablement conscience de sa nature propre, de sa liberté... lorsqu'il parvientà sentir et à penser tout en laissant ses sens remplir leurs fonctions pratiques...il fait l'expérience intime de la liberté la plus parfaite et du plus jubilatoire sentiment de puissance ${ }^{26}$.Ainsi, ce qui formera les liens entre les individus au sein d'un orchestre, ce sera autre chose que le verbe : un langage sensoriel, symbolique et non une forme communicative ${ }^{27}$ où chacun est obligé de faire silence et de déployer une attention soutenue pour pouvoir écouter l'autre. Or, écouter n'est autre qu'entrer dans le son et "se changer soi-même en son en existant en lui ${ }^{28}$ et dans les silences qui le produisent (avant et après son émission). Ainsi définie comme l'ensemble formé par les sons et les silencesappelés mapar le compositeur japonais Toru Takemitsu (1930 - ), la musique est le produit d'un silence et y retourne ; et c'est dans ces $m a$ « inquantifiables et tendus dynamiquement... emplis d'innombrables sonorités ${ }^{29}$ que se crée la véritable musique, vivante. Saisir ces espaces - temps entre les gens et la musique est le but de toute pratique collective qui mobilise avant tout cette écoute à la fois sensorielle et intuitive de tout le corps que nous savons mobiliser parfois au quotidien dans notre relation aux autres et au monde, sans y faire attention. Ainsi, celui qui fait l'expérience de l'orchestre fait l'expérience d'un temps musical qui le renvoie à un temps vécu pour soi-même et partagé avec d'autres ; la musique étant éprouvée dans ce jeu comme une succession discontinue d'instants sonores, situés hors du temps chronologique, elle libère l'individu plongé au sein d'un milieu bienveillant, au sens mésologique du terme.

23 La notion de jeu comme métaphore de la création est omniprésente dans la mythologie. La création du monde y est expliquée à partir de divinités qui, à travers leurs activités artistiques (danse, théâtre, musique...) créent l'univers. Il est à noter, à ce sujet, que le terme sanscrit pour dieu (deva) provient de la racine div qui signifie « jouer ».

24 Donald Woods Winnicott,Lénfant et le monde extérieur, le développement des relations.(Paris : Payot, 1972), 125.

25 DonaldWoods Winnicott,Conversations ordinaires. (Paris, Gallimard, 1988. [1986]).

26 Novalis, Les Disciples de Saïs, chap. II, Werke.(Munich : Beck 1981), 120.

27 Françoise Escal, Espaces sociaux, espaces musicaux.(Paris : L'Harmattan, 2009), 197.

28 ToruTakemitsu, cité par Alain Poirier, ToruTakemitsu.(Paris : Michel de Maule, 1996), 63-64.

29 Kimura Bin, L’Entre, Une approche phénoménologique de la schizophrénie.(Grenoble : Million, 2000), 57. 


\section{Le Milieu}

À la suite du philosophe et géographe Augustin Berque (1941- ), le fondateur de la mésologie (ou étude des milieux humains), je distingue l'environnementdumilieu. Toujours à construire, le milieu se définit comme "l'ensemble des relations éco-techno-symboliques que l'humanité crée à partir d'elle-même et de la matière première qu'est l'environnement $»^{30}$. Cela signifie que nous sommes reliés au monde d'une façon dynamique, dans un va-et-vient incessant,par les sens, la parole et l'action. Or, ces relations de réciprocité,toujours à créer, au sein d'un milieu (celui de l'orchestre en particulier) ne sont pas mesurables: elles relèvent de l'art dont l'étymologie (AR) donne l'idée de jointure, d'articulation entre deux choses ou entre deux êtres ; ce que résume le trait d'union dans l'orthographe de notre langue ${ }^{31}$. Dans la perspective qui est la nôtre, l'art consiste à créer par la musique et la pratique d'orchestre, un milieudistinct de l'environnement carcéral, qui puisse service demédiation entre les êtres et ouvrir un monde ${ }^{32}$.Dans ce milieu humain (c'est-à-dire dynamisé par des relations réciproques) et concret de l'orchestre,il est possible de croître ensemble comme l'indique l'étymologie du mot (concret : cum crescere). Par cette forme d'existence en relation ${ }^{33}$, il s'agit de déployer des capacités d'attention, base de toute éducation, indissociable d'un milieu confiant, qui permette à tous de se répondre et à chacun de répondre au monde et $d u$ monde. Dans cet espace commun, créé entre des personnes inconnues et responsables au sens qui vient d'être donné, peut alors se former une "pluralité $d$ 'individus singuliers »(Arendt) soutenus par une dynamique commune ; ce qui est la définition même de la politique qui prend naissance, selon Hannah Arendt dans l'espace-entre-les-hommes et se constitue comme relation dans cet espace intermédiaire. En élaborant un sens commun par et pour les citoyens,l'orchestre acquiert ainsi une fonction politique,dans le contexte de la prison qui vise au contraire à séparer les êtres.

Parmi eux, certains se sentent coupés du monde et de leurs semblables, ayant perdu le lien avec la vie. Le psychiatre musicien Kimura Bin, utilise ainsi la musique pour recréerce lien et faire sentir cette intuition de la vie par la pratique musicale collective qui favorise les interactions auprès d'individus schizophrènes. Car la vie on la ressent, on ne saurait la connaître ${ }^{34} \mathrm{et}$ les mots sont impuissants à donner l'idée immédiate de cette vie. Or, la musique émane de l'acte de vivre ;elle est (comme la danse) un jaillissement de la vie sous sa forme primitive et originelle $e^{35}$, autrement dit un rythme libre et singulier (et non pas une «mesure ») que chacun peut s'approprier dans son jeu. Considérée comme

30 Augustin Berque,Glossaire de mésologie. (Paris : éoliennes, 2018), 26. L'auteur distingue non seulement le milieu (à créer) de lenvironnement (le donné brut) mais également la mésologie (étude des milieux humains) de lécologie, centrée sur l’environnement. Historiquement, cette distinction est apparue en biologie chez J-V. Uexküll (1864-1944) et en philosophie chez Watsuji Tetsurô (1889-1960).

31 Augustin Berque, « De milieu en mésologie. L'art de Didier Rousseau-Navarre ». Didier Rousseau-Navarre, Les graines de l’art. La sculpture mésologique.(Paris : Le livre d’art, 2016), 7.

32 Martin Heidegger, «L'origine de l’ouvre d’art ». Chemins qui ne mènent nulle part. (Paris : Gallimard, 1986 [1962]), 49-50 : « Sur la terre et en elle, l'homme historial fonde son séjour dans le monde. Installant un monde, l’euvre fait venir la terre ».

33 Non seulement la relation nous précède mais elle est vitale pour lêtre humain comme lont démontré John Bowlby et René Zazzo dans la théorie de l'Attachement.cf. Le colloque sur l'Attachement, organisé par E. Zazzo, (Paris : Delachaux et Niestlé), 1979.

34 Kimura Bin, L'Entre.op., cit., 18

35 Kimura Bin, L'Entre, 34. 
une activité vitale(et à ce titre, aussi fondamentale que de manger, de dormir, de se reproduire ${ }^{36}$ selon Kimura Bin), la musique permet de retrouver un statut de sujet, en parole et en acte, relié à la réalité extérieure comme à son propre fond vital : c'est sous ce double rapport seulement qu'il devient unsujet vivant et agissantcapable de sentir et donc de penser. Or, nous ne vivons que par le maintien de cette relation vitale qui échappe à la mesure,dans le sentir et dans l'agir que le travail musical accompli au sein de l'orchestre nous fait éprouver. Cette éducation sensorielle s'avère essentielle au quotidien dans les rapports humains ; car apprendre à sentir c'est non seulement apprendre à vivre ensemble mais aussi à penser.

\section{Restaurer la capacité de sentir et de penser}

Si l'on admet que la capacité de sentir est indissociable de la capacité de penser $^{37}$ (comme l'a démontré le neurologue Antonio Damasio, pionnier dans la découverte de ce lien entre émotion et cognition, ou émotion et créativité38), la musique a un rôle essentiel à jouer dans nos sociétés, offrantune « invitation pour un voyage commun vers des destinations personnelles ${ }^{39}$. Chez des êtres dont les visages, impassibles et fermés semblent avoir perdu la capacité même de sentir c'est-à-dire, ce qui faisaient d'eux des êtres humains, il fut particulièrement gratifiant tout au long de ces années, d'observer leur transformation et voir ces visages sourire en s'ouvrant progressivement à la musique et aux autres pendant les séances. Comment mesurer celascientifiquement? Ce liant que nous avons établi à travers la musique autour de la personne détenue, par les sens, les silences et les sons, les regards et les gestes, et dont les résultats sont visibles sur les corps (à travers les sourires notamment) est hors de portée de la science comme de tout contrôle. Car ce qu'il y a entre les gens dans la vie comme dans la musique, relève de la sensibilité, fruit de la relation et de l'attention portée à chacun et à autrui. Ce qui n'est autre qu'une forme de soin, engageant la responsabilité de chacun au milieu de l'orchestre, devenu un lieu d'éducation à l'attention, une compétence vitale mobilisant avant tout la capacité de sentir. Cela fait de l'éducation un art de l'attention comme l'a exprimé l'anthropologue musicien Tim Ingold ${ }^{40}$ et une pratique des langages sensoriels.Cetteattitude qui consiste à écouter avec tout son corps pour rencontrer les choses et les êtres, fait déployer tout un champ de résonances et de réciprocitéset donne la joie de jouer. Celle-ci est fondamentale car elle s'oppose à la tristesse et l'indifférence qui coupent les sujets d'eux-mêmes et de leur potentiel de créativité comme le faisait remarquer le philosophe Spinoza (1632 - 1677) pour qui un tyran a intérêt à entretenir la

36 Ibid.

37 Donald Woods Winnicott, Conversations ordinaires, (Paris : Gallimard, 1988. [1986]). " Quand on lit des témoignages d'individus qui ont passé toute leur existence dans les camps de concentration ou encore qui ont subi leur vie durant, des persécutions politiques, ...seules quelques-unes de ces victimes parviennent à rester créatives tous les autres continuent d'exister mais ne vivent pas, ... Sans doute ont-ils perdu ce qui faisaient d'eux des êtres humains » : la créativité, fruit de la capacité d’éprouver, de sentir ». Je souligne.

38 Né à Lisbonne en 1944, il est le directeur de 1>Institut pour l’étude neurologique de l>émotion et de la créativité de l’Université de la Californie méridionale depuis 2005, après avoir été le directeur du département de neurologie de l'Université de l'Iowa pendant dix-huit ans.

39 Gilles Boudinet. De l'universel en musique. Fugues et variations d'un savoir.(Paris : Publi sud, 1995).

40 Tim Ingold, L'anthropologie comme éducation (Rennes : PUR, 2018). Dans cet ouvrage, l'auteur prend l'art comme modèle d'éducation pour l'anthropologie. 
tristesse pour mieux manipuler ses sujets ${ }^{41}$. Penser le sujet vivant et en relation de réciprocité avec un milieu qui le crée autant qu'il le crée, implique une nouvelle anthropologie où le sujet,jamais "fixé » dans une identité, est conçu comme point de contact entre l'organisme et le monde. Telle est l'hypothèse sur laquelle nous nous appuyons pour affirmer l'idée qu'un individu ne peut pas être réduit à ce que l'on dit de lui et qu'une identité n'est jamais donnée. Seul existe le processus jamais achevé de l'identification ${ }^{42}$ en relation avecun milieu toujours changeant dont il dépend largement.Ainsi, « la délinquance » n'est pas un état permanent ; le processus de sortie existe aussi (la désistance). Mais si tout être humain a la responsabilité de transformer en acquisition active la passivité d'une identité reçue pour conquérir sa liberté, cette mutation ne peut s'accomplir sans le soutien d'une société qui se montrera accueillante et conviviale.

\section{CONCLUSION}

\section{L'orchestre comme milieu convivial}

L'orchestre a été ici conçu comme une mini-société où la réussite s'accomplit collectivement, pouvantapporter des éléments de réponse à la question de la réinsertion des personnes détenues depuis la prison même. Dans cette perspective, l'orchestre participatif que nous avons créé dans d'autres milieux que l'environnement carcéral, doit être reconnu d'utilité publiquecar il contribue à améliorer les conditions de vie des personnes en favorisant le convivialisme ${ }^{43}$, devenu aujourd'hui une philosophie nécessaire au vivre-ensemble.

La situation dans laquelle nous nous trouvons,nous oblige à nous entendre au niveau mondial sur les valeurs essentielles à défendre pour la survie, tant matérielle que morale, de l'humanité et sur ce que signifie ce mot d'humain. L'appât du gain, le culte du moi et l'indifférence qui constituaient jusqu'à présent la norme sociale ne sont pas propices à la construction d'un monde reposant sur d'authentiques relations entre les gens (autres que l'argent et le marché) et dans lequel il serait possible de «s'opposer sans se massacrer » (M. Mauss). Tel est le constat du mouvement International Convivialiste ${ }^{44}$ dont les auteurs ont repéré dans leur Second manifeste les deux principaux fléaux qui menacent aujourd'hui l'humanité: l'explosion mondiale des inégalités dues à une sur-consommation illimitée, et d'autre part les menaces climatiques aux conséquences à la fois économiques, sociales et humaines croissantes. Face à ces dangers, l'urgence est de coopérer afin de repenser notre existence sur de nouvelles bases, en pratiquant l'art de la convivence ou art de vivre ensemble (con-vivere) qui met au centre la

41 Baruch Spinoza,Ethique,I à V, CEuvres, (Paris: Flammarion, 2008), 97-535. Ce philosophe montre que la recherche de la joie comme manière dêtre et de vivre, confère à la liberté et la sagesse et devient un projet politique en s'opposant à la tristesse entretenue par les tyrans.

42 Jacques Derrida, Le Monolinguisme de l’autre.(Paris : Galilée, 1996), 53.

43 Cemouvement est né en France à l’initiative de Alain Caillé, Manifeste Convivialiste. Déclaration d'interdépendance. (Paris : Le Bord de l'eau, 2013). Second Manifeste Convivialiste International. Pour un monde post-néolibéral. (Paris : Actes Sud, 2020). Les auteurs cherchent à élaborer une pensée et une intelligibilité du monde afin de se mettre d'accord à léchelle mondiale, sur les valeurs essentielles de survie (matérielle et morale) de l'humanité, s'interrogeant en particulier sur le sens du mot « humain » aujourd'hui.

44 Internationale Convivialiste. Second Manifeste convivialiste. Pour un monde post-néolibéral.(Paris : Actes Sud, 2020). 
relation entre les humains; dans cette philosophie, il s'agit de prendre soin des autres et de la nature selon une éthique déclinée en cinq principes: commune naturalité, commune humanité, commune socialité, légitime individuation et opposition créatrice ; ces deux derniers principes qualifient selon nous le travail artistique, celui de l'orchestre en particulier, qui permet à chacun de développer son individualité et ses capacités propres au service de la collectivité tout en permettant de se différencier (par la pratique d'un instrument singulier) et de s'opposer de manière bienveillante, ce qui est au fondement de toute véritable démocratie comme de l'orchestre.

\section{BIBLIOGRAPHIE}

Añaños-Bedriñana, F.T., coordinadora. Las mujeres en las prisiones. La Educación social en contextos de riesgo y conflicto Barcelona: Gedisa, 2010.

Añaños-Bedriñana, F.T., \& García-Vita, M.M. (2017. ¿Desarrollo humano en contextos punitivos. Análisis Socioeducativo desde las vulnerabilidades sociales y el género. Revista Criminalidad, 59 (2): 109-124.

Andrieu,Michaël. De la musique derrière les barreaux. Préface de Philippe Combessie. (París : L'Harmattan, 2005).

Arendt, Hannah. Qu'est-ce que la politique ? Texte établi par Ursula Ludz, París : Seuil, 1995.

Beccaria, Cesare. Des délits et des peines. Traduction de Maurice Chevallier. Préface de Robert Badinter. Paris : Flammarion, 1991, 23. [Genève 1965].

Berque, Augustin.Glossaire de mésologie. Paris : éoliennes, 2018.

Berque, Augustin. " De milieu en mésologie. L'art de Didier Rousseau-Navarre », Didier Rousseau-Navarre, Les graines de l'art. La sculpture mésologique. Paris : Le livre d'art, 2016.

Bowlby, John et Zazzo, René. L'Attachement, Paris : Delachaux et Niestlé, 1979.

Boudinet, Gilles. De l'universel en musique. Fugues et variations d'un savoir. Paris : Publi sud, 1995.

Caillé, Alain et Chanial, Philippe. Du convivialisme comme volonté et comme espérance.Revue du Mauss $\mathrm{N}^{\circ} 43$ (premier semestre 2014).

Chantraine,Gilles et Epstein,Renaud. «Des quartiers relégués au contrôle des prisons : l'action libre d'un haut fonctionnaire. Entretien avec Jean-Marie Delarue » Prison : l'idéologie de l'enfermement, Revue Mouvements No 88. (2016) :136-149.

Chantraine,Gilles et Sallé Nicolas. Eduquer et punir. Travail éducatif, sécurité et discipline en établissement pénitentiaire pour mineurs. Vol : 54 . No3 (2013).

Chauvenet, Antoinette, Rostaing, Corinne et Orlic Françoise. La Violence carcérale en question

(Paris : PUF, 2008).

Collectif de l'atelier « Prison ». Parce qu'ils sortiront un jour. L'insertion postpénale des personnes détenues, un défi citoyen.(Association Demosthène, Caen : 2016).

Cholet, Didier (dir), Les nouvelles prisons. Enquête sur le nouvel univers carcéral français. Rennes : PUR, 2015.

Delarue, Jean-Marie, «L'Horreur carcérale. Continuité et discontinuité de la condition pénitentiaire ", in Caillé, Alain et Fixot, Anne-Marie. Sortir de la prison. Entre don, abandon et pardon. Revue du Mauss No 40 (second semestre 2012) : 73-102.

Derrida, Jacques. Le Monolinguisme de l'autre. Paris : Galilée, 1996.

Escal, Françoise. Espaces sociaux, espaces musicaux. Paris : L'Harmattan, 2009.

Heidegger, Martin. «L'origine de l'œuvre d'art ». Chemins qui ne mènent nulle part. Paris : Gallimard, 1986 [1962].

Huizinga, Johan. Homo ludens, Essai sur la fonction sociale du jeu.Traduit du néerlandais par Cécile Seresia. Paris : Gallimard, 1988.

Ingold, Tim. L'anthropologie comme éducation. Rennes : PUR, 2018.

Kimura, Bin. L'Entre.Une approche phénoménologique de la schizophrénie. Grenoble : Millon, 2000. 
et d'éducation au sein des prisons

Lassus, Marie-Pierre (dir.).Le Jeu d'Orchestre. Recherche-action dans les lieux de privation de liberté.PUL: 2015.

Lassus, Marie-Pierre. Le non-savoir, paradigme de connaissance, Louvain-París: EME,

L'Harmattan, 2019.

Lassus, Marie-Pierre. «La musique ou la joie» Revue internationale d'art et d'artologie. $\mathrm{N}^{\circ} 2$ (2019) : 39-55.En ligne https://www.effet-de-vie.org

Premier Manifeste Convivialiste. Déclaration d'interdépendance. París: Le Bord de l'eau, 2013.

Second Manifeste convivialiste. Pour un monde post-néolibéral. París: Actes Sud, 2020.

Mauss, Marcel.Essai sur le don : forme et raison de l'échange dans les sociétés archaïques. Sociologie et anthropologie. Paris : PUF, 1950.

Milhaud Olivier et MorelleMarie. «La prison entre monde et antimonde ».Géographie et culture. (57), Paris : L'Harmattan, 2006.

Mora Aragon, Kleiberth Lenin, Trascendencia de la conducta a través de la práctica orquestal en ambientes poco gratificantes. Informe técnico (2007-2014), 5.

Münch, Marc-Mathieu. L'effet de vie. Le phénomène " art ». Revue en ligne https://www.effet-de-vie. org

Novalis.Les Disciples de Saïs, chap. II, Werke, Munich: Beck 1981.

Poirier, Alain.ToruTakemitsu. Paris : Michel de Maule, 1996.

Portelli, Serge et Chanel, Marine. La vie après la peine. (Paris :Grasset, 2014).

Rostaing, Corinne. La Relation carcérale. Identités et rapports sociaux dans les prisons de femmes. (Paris: PUF, 1997).

Siganos, Florine. L'action culturelle en prison. Pour une redéfinition du sens de la peine. Paris: L'Harmattan, 2008.

Spinoza, Baruch Ethique, I à V, CEuvres, (Paris: Flammarion, 2008), 97-535.

Wacquant, Loic, «Les geôles du précariat » Comment supporter la prison ?Revue Vie Sociale et Traitement $\mathrm{N}^{\circ} 124$ (4 ${ }^{\mathrm{e}}$ semestre 2014).

Wilde, Oscar. «De Profondis ». CEuvres. (Paris: NRF Gallimard, 1996).

Winnicott,Donald Woods.L'enfant et le monde extérieur, le développement des relations. Paris: Payot, 1972,

Winnicott,Donald Woods.Conversations ordinaires.Paris: Gallimard, 1988. [1986].

Cómo citar:

Pierre Lassus - Marie. "L'orchestre : un milieu de convivence et

d'éducation au sein des prisons". Revista Historia de la Educación

Latinoamericana. 22 No. 35 (2020): 103-117

https://doi.org/10.19053/01227238.11921

(c) (i) @(@) Esta obra está bajo una licencia Creative Commons.

Reconocimiento-No Comercial-Sin Obra Derivada 2.5 Colombia. 
